\title{
Cooperative Learning: Value-Added To Operations Management
}

Brian Fitzpatrick, Rockhurst College, USA

Shahid I. Ali, Rockhurst College, USA

\begin{abstract}
Integrating cooperative learning techniques with information technology and applying it to the field of Production and Operations Management (P/OM) will help to investigate how ComputerSupported Cooperative Learning (CSCL) can enhance learning performance of students. Research in CSCL provides evidence that collaborative communication technology can be a better mechanism to add value to education. Most studies, however, have examined learning performance only during the middle and end of the learning process. Thus, it is not clear how information technology can continuously facilitate and improve student performance and learning experience over time. The purpose of this research is to examine how information technology, when applied to communication medium, can facilitate the learning process in the field of production and operations management. This study compared traditional, face-to-face cooperative learning environments to computer-supported cooperative learning environments for differences in students' learning performance and group member satisfaction.
\end{abstract}

Keywords: Computer-Supported Cooperative Learning (CSCL); Integrating Cooperative Learning Techniques (ICLT); Face-to-Face Learning (FtFL); Computer-Mediated (CM); Production and Operations Management (P/OM)

\section{INTRODUCTION}

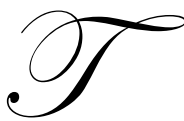

oday's business environment is very challenging in nature. Students need to integrate and apply knowledge from various domains. In order to integrate and apply knowledge, it is necessary for them to retain knowledge from different disciplines. The problem of retaining knowledge could be addressed if students are exposed to an environment where they can learn from themselves. One way to address the problem is to seek help from information technology. Integrating cooperative learning techniques with information technology and applying it to the field of production and operations management (P/OM) will help to investigate how Computer-Supported Cooperative Learning (CSCL) supports the understanding of P/OM materials from the students' perspective. CSCL is the use of information technology in instructional settings and is designed to facilitate greater learning, based on group cooperation (Alavi, 1994; Brandon \& Hollingshead, 1999). Cooperative learning is a philosophy that enables students to learn from one another. It is an instructional method that creates an environment to challenge students and develop better decision-making skills. Drawing from the success of more traditional Face-to-Face Cooperative Learning (FTFCL) environments, CSCL is intended to form a synergic collaboration between information technology and cooperative learning. Previous studies show that cooperative learning facilitates increased activeness on the students' part and improves information exchanges. Symons and Symons (1995) have made a small effort in terms of introducing a rationale for incorporating a cooperative learning philosophy into production/operations management instructions.

Literature has supported the positive outcome of cooperative learning in different disciplines (Symons \& Symons, 1995; Maier \& Keenan, 1994). However, it is yet to be established what will be the outcomes if the concept is applied to differentiate between FTFCL \& CSCL in the field of production and operations management. Since operations management is very quantitative in nature, the purpose of the research is to see the effect of information technology combined with cooperative learning in two different mediums and observe the effect of the learning process from a decision-making perspective. The research has examined how computer-mediated (CM) collaborative technology facilitates individual and group learning as well as promotes satisfaction with this type of 
learning environment over the course of a semester. The present research is different from the previous studies as it is longitudinal in nature, with continuous observations of performance and satisfaction made throughout the entire semester. Previous studies have generally observed learning groups only during the middle and end of the school semester.

\section{BACKGROUND}

Cooperative learning is defined as a group process in which students acquire knowledge, skills, and attitudes by actively participating in solving academic problems (Alavi, 1994; Brandon \& Hollingshead, 1999). Compared to the traditional instructor-centered lecture method, the use of collaborative learning methods can significantly promote positive academic and affective outcomes among students (Brandon \& Hollingshead, 1999). Positive effects of cooperative learning may include improvements in academic achievement, the generation of more ideas and solutions, higher self-esteem, more positive attitudes toward school, as well as promoting the ability to work cooperatively (Slavin, 1991). Research in CSCL is intended to amplify these benefits with the use of information technology (Alavi, 1994), while eliminating common time and space constraints that are found in traditional face-to-face (FtF) classroom settings (Brandon \& Hollingshead, 1999). The effects of CSCL have been studied from different perspectives. It has been studied from the perspective of distance learning (Webster \& Hackley, 1997). It has also been investigated in the small group decision-making process (Olaniran, 1994). As evident, the application of CSCL has been applied from various perspectives. However, no effort has been made to apply the concept of CSCL to the field of operations management. The main motivation of this study was to investigate how CSCL could benefit the field of operations management. Business educators have explored the techniques of FTFCL vs. CSCL to their respective disciplines with advantages in one medium over the other (Leidner \& Jarvenpaa,1995; Alavi,1994; Olaniran,1994; Galbraith, 1994; Taylor,1991). It is very obvious from these studies that the choice of one medium over the other is not an easy task. The purpose of using technology in a cooperative learning environment is to facilitate the learning process. The question addressed is how technology in a cooperative learning environment helped the learning process in the field of operations management. The focus of the research was to investigate whether learning performance and learning satisfaction can be achieved better in a CSCL environment vs. a FTFCL environment when applied to the field of operations management. Since technology was used as a delivery vehicle, the question arises what technology features was best used to deliver the content. Do students learn better operations management concepts using technology versus not using technology in a cooperative learning environment?

The research examines how CMCL facilitates individual and group learning as well as promotes satisfaction with this type of learning environment over the course of a semester.

\section{THEORETICAL FRAMEWORK: HYPOTHESES}

The theoretical foundation of this research is adapted from the descriptive model of computer-supported cooperative learning (Brandon \& Hollingshead, 1999). This model suggests that in addition to being subject to various contingent factors, the effectiveness of learning is bounded by the type of technology and style of instruction. This study was intended to examine how collaborative technology facilitates learning by investigating the effects of communication media on the performance of learning over time. The performance of learning consists of two parts: academic and affective outcomes. Academic outcomes refer to the mastering and perceived mastering of learning materials, while affective outcomes refers to subjectively assessed satisfaction with the learning process. (Yazici 2004) did find that students will perceive that collaborative learning, enhances their critical thinking skills in the study of operation management problems.

Academic outcomes are typically the focus of evaluation when comparing $\mathrm{CM}$ to $\mathrm{FtF}$ learning environments (Hiltz, 1993). The majority of previous research (Alavi, 1994; Althaus, 1997; Hiltz, 1993; Hiltz \& Wellman, 1997) suggests that students in CM environments tend to have better mastery of learning materials. Research by Alavi (1994) and Althaus (1997) further indicate that students in CM groups have significantly higher performance for final exams than students in FtF groups, but no significant differences in midterm exams. Possible explanation for this finding can be found in communication research. Hollingshead, McGrath, and O'Connor (1993) assert that the performance of group members working together is affected by the level of their familiarity with the 
communication medium. This suggests that students in CM groups would not perform as well as their counterparts in FtF groups until they become familiar with the medium. As the semester proceeds, students in CM groups should become more proficient with the use of the CM medium and, consequently, their performances should improve. This proficiency might be further strengthened, as suggested by Adaptive Structuration Theory (Poole \& DeSanctis, 1989), by students' adaptation of technology structures. It is believed this proficiency, in turn, will improve students' learning performance over time.

We summarize our hypotheses regarding learning performance as follows:

H1: Learning performance differences between CSCL and FTFCL groups will be consistently significant during the entire semester.

In addition to objective learning performance, perceived learning performance is another area considered by researchers in this area. Studies by Alavi (1994) and Althaus (1997) found that computer support has a significant effect on perceived learning performance because students in CM groups felt that they were exposed to diverse perspectives and various ideas. However, as found by many researchers in the area of CM communication, in early sessions this advantage might be offset by the fact that CM participants need more time to finish their assignments (Bordia,1997). Consequently, the perception of better learning performance is gradual.

H2: $\quad$ Perceived learning performance differences between CSCL and FTFCL groups during the entire semester will be consistently significant.

Students in FtF environments tend to be more satisfied with the process than students in CM environments (Benbunan \& Hiltz, 1999). The lower level of satisfaction in CM groups might be attributed to the lack of visual cues, such as facial expression and members' unfamiliarity with the medium (Hollingshead, McGrath, and O'Connor,1993). However, members in CM environments tend to indulge in more task-oriented conversation (Bordia,1997), thus promoting greater achievement, which in turn may positively affect satisfaction with the learning process (Kahai \& Cooper, 1999). Additionally, as suggested by the Adaptive Structural Theory (Poole \& DeSanctis,1989) students in CM groups over time might adapt structures in the medium to fit the context of their learning environment in a way that best suites them. As a result, the difference in satisfaction between $\mathrm{CM}$ and FtF groups may diminish over time.

H3: No consistently significant differences in group member satisfaction will be found between CSCL and FTFCL groups for the entire semester.

\section{METHODS}

\section{Participants}

One hundred and nineteen undergraduate students enrolled in three sections of a one semester-long course in Production and Operations Management participated in this study. Students were randomly assigned to either the CMCL condition or the FTFCL condition. Each condition consisted of 20 groups. Each group consisted of three, randomly assigned members (except one CMCL group which had 2 group members) - 59 students in the CSCL condition and 60 students in the FTFCL condition. All three sections were taught by the same instructor with the same textbook, teaching materials, coverage, examinations, and requirements. Participation in the study was one of two options available to fulfill the partial requirement for the course.

\section{Research Design}

The dependent variable - learning effectiveness - was comprised of two components: the actual learning of the students and their subjective evaluation of the learning process. The academic learning performance was measured by students' quiz scores. Perceived learning performance was measured by items adopted from previous research. The perceived learning performance measured the subjects' estimate of how much they learned within different environments. 
The subjective, evaluative part of the learning process refers to students' satisfaction with the groupmeeting environment. The satisfaction measure used in this study was derived from Olaniran, which measured group satisfaction within $\mathrm{CM}$ and $\mathrm{FtF}$ environments.

\section{Learning Cases}

To assess the degree of learning for both conditions in this study, six cases were used. These cases were taken and modified from the textbooks of Production and Operations Management by Stevenson and by Render and Heizer. Each case described a scenario in which the decision-maker needed to make a plan or reach a decisionwhich would be considered a decision-making task according to McGrath's Typology of Tasks.

The cases included the background of the scenario, the problems and/or the opportunity the decision maker faces, and the requirements for an acceptable and feasible solution. Also, two or three questions were included in each case to guide students' discussion. Those questions were general enough for team members to propose various ideas that were directional in nature in that multiple solutions were possible.

\section{Procedure}

The experiments were conducted biweekly. No specific job divisions were assigned to the individual team members. The meetings of CSCL groups were held in a computer laboratory with $24 \mathrm{PCs}$, each of which was equipped with a synchronous communication program (ICQ ${ }^{\mathrm{TM}}$ ). Subjects in the CM groups were introduced to the operation of ICQ and were briefed as to the purpose and procedures of the research.

The collaborative learning process used in this study was adapted from Student Team-Achievement Division (STAD). The meetings lasted fifty minutes and proceeded as follows: Each team member studied the case on his/her own for fifteen minutes and took notes of his/her ideas about the case. In the following thirty-five minutes, team members discussed the case, exchanged their ideas, and reached their conclusion about the case. At the end of each experiment, CSCL groups were required to save their discussion, including discussion threads and conclusions. No specific instructions regarding task allocation were given. Thus, team members might take turns to write up the report.

\section{RESULTS AND DISCUSSION}

Multivariate repeated measures analysis of variance was performed on three dependent variables: performance $(\mathrm{H} 1)$, perceived performance $(\mathrm{H} 2)$, and satisfaction (H3). A MANOVA repeated measures procedure was used to analyze the data. Where interaction effects were found, further analysis was performed on the dependent variables in the early or later sessions. The results of analyses were presented in this section and where appropriate.

\section{PERFORMANCE}

Learning performance was measured with quizzes consisting of multiple-choice questions. The questions were to test students' mastery and application of concepts used in the case.

H1 predicted that learning performance differences between CSCL and FTFCL groups during the entire semester would be consistently significant. This hypothesis was supported $[\mathrm{F}(5.34)=12.66, \mathrm{p}<.001]$. This is consistent with previous studies which have found little differences in performance between CM and FTFCL groups in the early stages of group development. However, it was also hypothesized that CSCL groups, after adjusting to their environment, would increase their level of performance and outperform the FTFCL groups in later sessions. Despite this outcome, except for the last session, the CSCL groups did out-perform their counterpart in the FTFCL environment. Yet, as seen in Figure 1, there was little consistency in performance between the CSCL and FTFCL groups. There are two possible explanations for the unexpected finding. One possibility is that, as Gersick suggests, groups will develop following a punctuated equilibrium model. That is, groups in this study alternated relatively stable periods of activity. However, transitions from one period to another were punctuated by significant changes in group-member behavior, triggered by such things as the realization of time pressure and deadlines. The poor 
performance of both groups in the third session and the rebound in the fourth session might be due to students' awareness of approaching the end of the semester. Another possibility is that while the measurement instrument was designed to evaluate students' mastery of concepts and principles, the nature of several topics was relatively quantitative. In other words, the nature of the topic had greater effect on the performance than the medium itself.

Figure 1: Profile Plot of Learning Performance over Time

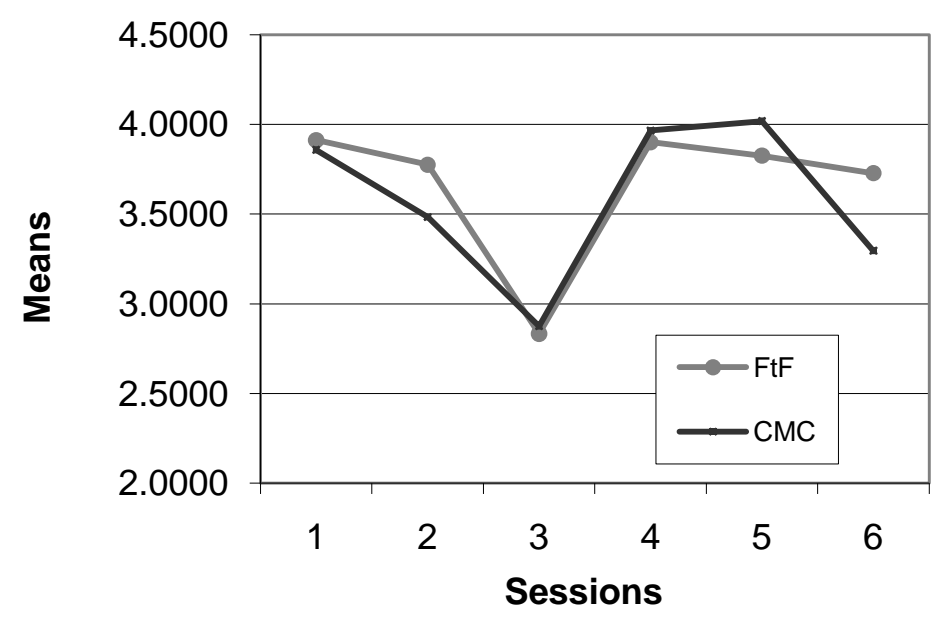

\section{PERCEIVED PERFORMANCE}

Perceived performance was measured after each group session, using a three-item semantic differential scale with a reliability of (Cronbach's $\alpha$ ) of 0.89 . H2 predicted that perceived learning performance differences between CSCL and FTFCL groups during the entire semester would be consistently significant. This hypothesis was supported $[\mathrm{F}(5,34)=2.729, \mathrm{p}<0.04]$.

As the profile plot in Figure 2 shows, perceived performances in both the CSCL and FTFCL groups are generally increasing with FTFCL groups, for the most part, having higher levels of perceived performance. This suggests that over time, both groups become accustomed to their learning environment and consequently develop a greater confidence in their performances. However, it is believed that the FTFCL groups start off with, and generally continue to have, greater perceptions of learning performance because of the more immediate feedback, as well as greater range of cues that are associated with $\mathrm{FtF}$ environments as opposed to $\mathrm{CM}$ environments. This result, however, is different from previous studies. The discrepancy might be caused by different levels of technological support and modes of communication between this study and others.

Figure 2: Profile Plot of Perceived Performance over Time

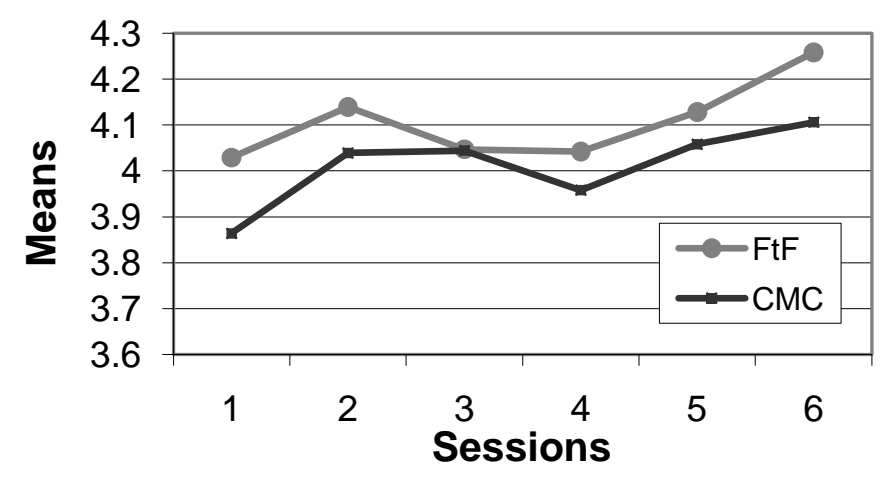


In the Alavi study, collaborative technology support of level 2 was used, while the present study adopted level 1 technology support. The novelty and sophistication of level 2 collaborative technology might significantly affect subjects' perception. Furthermore, asynchronous communication mode of the medium adopted in Althaus's study gave students more time to contemplate issues and others' perspectives before they responded. As a result, online subjects would perceive more positively their learning performance than their counterpart without computer support.

\section{SATISFACTION}

Satisfaction with the learning process was measured after each group session, using a seven-item semantic differential scale, adapted from Olaniran. A Cronbach Alpha Coefficient of 0.90 for the scale was obtained. H3 stated that no consistently significant differences in group member satisfaction would be found between CSCL and FTFCL groups for the entire semester, which was supported $[\mathrm{F}(5.34)=5.416, \mathrm{p}<.001]$.

The profile plot of satisfaction over time (Figure 3) indicates that the differences in satisfaction between groups were not consistently significant. However, after the second session, the FTFCL and CSCL groups had no consistent pattern. The cause for this discontinuity needs further investigation. Combined with the increasing pattern in the first three sessions, it seems fair to say that, in general, CSCL groups were gradually satisfied with the electronic medium, as suggested by Adaptive Structural Theory.

Figure 3: Profile Plot of Satisfaction over Time

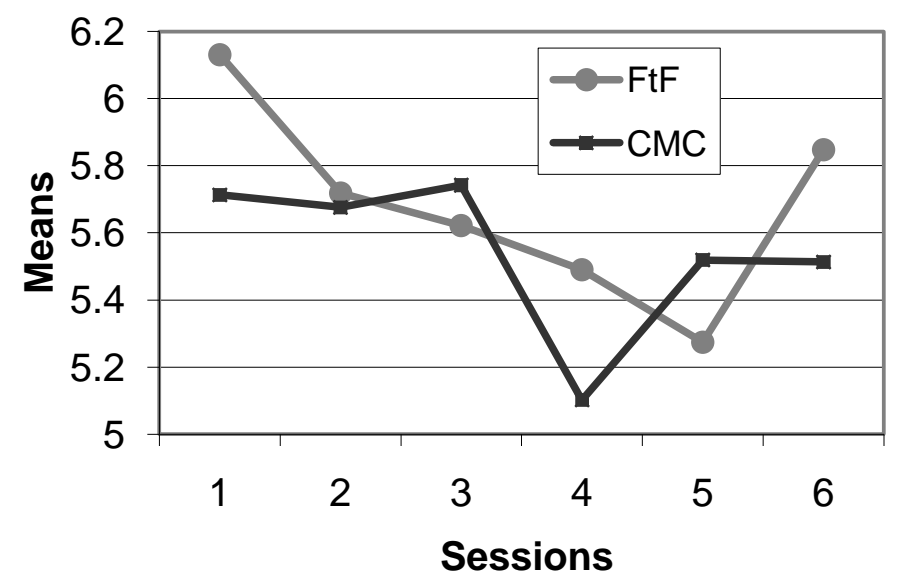

\section{CONCLUSIONS}

This study compared a traditional, $\mathrm{FtF}$ cooperative learning environment to a computer-supported cooperative learning environment to determine differences in students' learning performances. To do this, this study recurrently measured academic performance and subjective assessment of satisfaction with the learning environments during the course of one semester. The results showed that the learning performance differs across media over time. However, the difference in learning performance was not consistently significant. In general, FtF groups were more satisfied with the communication environment than their counterpart in the $\mathrm{CM}$ environment. Yet, this difference in satisfaction between two environments diminished over time. Finally, students in the FtF environment consistently perceived higher learning performance than CM groups. 


\section{AUTHOR INFORMATION}

Brian D. Fitzpatrick is a professor of finance for the Helzberg School of Management at Rockhurst University in Kansas City, Missouri. He earned his Ph.D. in Finance with a concentration in Investments from Saint Louis University. Dr. Fitzpatrick spent ten years in the Investment field including positions with two major Wall Street wire houses, as well as serving as senior vice president for both Financial Corporation of America and Commerce Bancshares. Brian has won the Rockhurst University's Teaching Excellence Award, and he has published in nine different fields.

Shahid I. Ali is an Associate Professor of Operations Management for the Helzberg School of Management at Rockhurst University in Kansas City, Missouri. He received his Ph.D. in operations management from Texas Tech University. Dr. Ali's primary research interests are in the areas of petroleum pipeline operations, information systems, and collaborative technology. He is a member of various professional societies and has published in Southern Business and Economic Journal, International Journal of Operations and Quantitative Management, Journal of International Technology and Information Management, Journal of Diversity Management and proceedings of Decision Sciences Institute.

\section{REFERENCES}

1. Alavi, Maryam. (1994). Computer-mediated collaborative learning: An empirical evaluation. MIS Quarterly, 18:2, June 1994, pp. 159-174.

2. Althaus, S. L. (1997). Computer-mediated communication in the university classroom: An experiment with on-line discussions. Communication Education, 46, 158-174.

3. Benbunan-Fich, R. \& Hiltz, S. R. "Educational Applications of CMCS: Solving Case Studies through Asynchronous Learning Networks.” Journal of Computer-Mediated Communication, 4:3, 1999, http://www.ascusc.org/jcmc/vol4/issue3/benbunan-fich.html.

4. Bordia, Prashant. (1997). Face-to-Face Versus Computer-Mediated Communication: A Synthesis of the Experimental Literature. The Journal of Business Communication, 34:1, pp. 99-120.

5. Brandon, David P., \& Hollingshead, Andrea B. (1999). Collaborative Learning and Computer-Supported Groups. Communication Education, 48, 109-126

6. Galbraith, J. R. (1994). Competing with flexible lateral organizations $\left(2^{\text {nd }}\right.$ ed.). Reading, MA: AddisonWesley.

7. Hiltz, Starr Roxanne. (1993). Correlates of Learning in a Virtual Classroom. International Journal of Man-Machine Studies, 39, 71-98.

8. Hiltz, Starr Roxanne, \& Wellman, Barry. (1997). Asynchronous Learning Networks as a Virtual Classroom. Communications of the ACM, 40:9, pp. 44-49

9. Hollingshead, Andrea B., McGrath, Joseph E., \& O'Connor, Kathleen M. (1993). Group Task Performance and Communication Technology: A Longitudinal Study of Computer-Mediated Versus Faceto-Face Work Groups. Small Group Research, 24:3, 307-333.

10. Kahai, S. S., \& Cooper, R. B. "The Effect of Computer-Mediated Communication on Agreement and Acceptance." Journal of Management Information Systems, 16:1, 1999, pp. 165-188

11. Leidner, Dorothy E., \& Jarvenpaa, Sirkka L. (1995). The use of information technology to enhance management school education: A theoretical view. MIS Quarterly, 19:3, Sep 1995

12. Maier, Mark H., \& Keenan, Diane. (1994). Teaching Tools: Cooperative Learning in Economics. Economic Inquiry, pp. 358-361.

13. McGrath, J. E., Groups: Interaction and Performance. Englewood Cliffs, NJ: Prentice-Hall, 1984

14. Poole, M. S. \& DeSanctis, G. "Understanding the Use of Group Decision Support Systems: The Theory of Adaptive Structuration.” In J. Fulk and C. Steinfield (eds.), Organizations and Communication Technology, Newbury Park, CA: Sage Publications, 1989, pp. 173 - 193.

15. Olaniran, B. A. (1994). A model of group satisfaction in computer-mediated communication and face-toface meetings. Behaviour \& Technology, 15, 24-36.

16. Render, B. \& Heizer, J. Production \& Operations Management. Englewood Cliffs, NJ: Prentice Hall, 1996

17. Slavin, R. E. Cooperative Learning: Theory, Research, and Practice. Englewood Cliffs, NJ: Prentice-Hall, 1990 
18. Slavin, Robert E. (1991, Feb.). Synthesis of Research on Cooperative Learning. Educational Leadership, pp. 71-82.

19. Stevenson, W. J. Production Operations Management. Publisher: Irwin McGraw-Hill, 2006

20. Symons, R.T. \& Symons, C.W. (1995). Proceedings of the twenty-sixth DSI Conference at Boston.

21. Taylor, W. (1991). The logic of global business. Harvard Business Review, 91-105.

22. Webster, J., \& Hackley, P. (1997). Teaching effectiveness in technology-mediated distance learning. Academy of Management Journal, 40(6), 1282-139.

23. Yazici, Hulya J. (2004). Student Perceptions of Collaborative learning in Operations Management Classes. Journal of Education of Business, November/December 2004, 110-118.

\section{NOTES}

